\title{
Systematic Review of Interventions to Reduce Urinary Tract Infection in Nursing Home Residents
}

\author{
Jennifer Meddings, MD, MSc ${ }^{1-3^{*}}$, Sanjay Saint, MD, MPH ${ }^{1,3}$, Sarah L. Krein, PhD, RN ${ }^{1,3}$, Elissa Gaies, MD, MPH ${ }^{4}$, Heidi Reichert, \\ $\mathrm{MA}^{1}$, Andrew Hickner, MSI ${ }^{1,5}$, Sara McNamara, MPH${ }^{6}$, Jason D. Mann, MSA ${ }^{1}$, Lona Mody, MD, MSc ${ }^{1,3,6}$
}

\begin{abstract}
${ }^{1}$ Department of Internal Medicine, Division of General Medicine, University of Michigan Medical School, Ann Arbor, Michigan; ${ }^{2}$ Department of Pediatrics and Communicable Diseases, Division of General Pediatrics, University of Michigan Medical School, Ann Arbor, Michigan; ${ }^{3}$ VA Ann Arbor Healthcare System, Ann Arbor, Michigan; ${ }^{4}$ St. Joseph Mercy Ann Arbor, Ann Arbor, Michigan; ${ }^{5}$ Cushing/Whitney Medical Library, Yale University, New Haven, Connecticut; ${ }^{6}$ Department of Internal Medicine, Division of Geriatric and Palliative Medicine, University of Michigan Medical School, Ann Arbor, Michigan.
\end{abstract}

BACKGROUND: Urinary tract infections (UTIs) in nursing homes are common, costly, and morbid.

PURPOSE: Systematic literature review of strategies to reduce UTIs in nursing home residents.

DATA SOURCES: Ovid MEDLINE, Cochrane Library, CINAHL, Web of Science and Embase through June 22, 2015.

STUDY SELECTION: Interventional studies with a comparison group reporting at least 1 outcome for: catheter-associated UTI (CAUTI), UTIs not identified as catheter-associated, bacteriuria, or urinary catheter use.

DATA EXTRACTION: Two authors abstracted study design, participant and intervention details, outcomes, and quality measures.

DATA SYNTHESIS: Of 5794 records retrieved, 20 records describing 19 interventions were included: 8 randomized controlled trials, 10 pre-post nonrandomized interventions, and 1 nonrandomized intervention with concurrent controls. Quality (range, 8-25; median, 15) and outcome definitions varied greatly. Thirteen studies employed strategies to reduce catheter use or improve catheter care; 9 studies employed general infection prevention strategies (eg, improving hand hygiene, surveillance, contact precautions, reducing antibiotics). The 19 studies reported 12 UTI outcomes, 9 CAUTI outcomes, 4 bacteriuria outcomes, and 5 catheter use outcomes. Five studies showed CAUTI reduction (1 significantly); 9 studies showed UTI reduction (none significantly); 2 studies showed bacteriuria reduction (none significantly). Four studies showed reduced catheter use (1 significantly).

LIMITATIONS: Studies were often underpowered to assess statistical significance; none were pooled given variety of interventions and outcomes.

CONCLUSIONS: Several practices, often implemented in bundles, such as improving hand hygiene, reducing and improving catheter use, managing incontinence without catheters, and enhanced barrier precautions, appear to reduce UTI or CAU$\mathrm{TI}$ in nursing home residents. Journal of Hospital Medicine 2017;12:356-368. (C) 2017 Society of Hospital Medicine
Given the limited number of geriatricians in the U.S., hospitalists commonly manage nursing home residents admitted for post-acute care. ${ }^{1-4}$ Urinary tract infection (UTI) is one of the most common infections in nursing homes, often leading to sepsis and readmission to acute care. ${ }^{5}$ Inappropriate use of antibiotics to treat asymptomatic bacteriuria is both common and hazardous to nursing home residents. ${ }^{6} \mathrm{Up}$ to $10 \%$ of nursing home residents will have an indwelling urinary catheter at some point during their stay. ${ }^{7-9}$ Residents with indwelling urinary catheters are at increased risk for catheter-associated urinary tract infection (CAUTI) and bacteriuria, with an estimated $50 \%$ of catheterized residents developing symptomatic CAUTI. ${ }^{5}$ While urinary catheter

\footnotetext{
*Address for correspondence and reprint requests: Jennifer Meddings, MD, MSC 2800 Plymouth Road, Building 16, Room 430W, Ann Arbor, Ml 48109-2800; Telephone: 734-936-5216; Fax: 734-936-8944; E-mail: meddings@umich.edu Additional Supporting Information may be found in the online version of this article.
}

Received: July 22, 2016; Revised: October 7, 2016; Accepted: October 18, 2016

2017 Society of Hospital Medicine DOI 10.12788/jhm.2724 prevalence is lower in nursing homes than in the acute care setting, duration of use is often prolonged. ${ }^{7,10}$ In a setting where utilization is low, but use is prolonged, interventions designed to reduce UTI in acutely ill patients ${ }^{11}$ may not be as helpful for preventing infection in nursing home residents.

Our objective was to review the available evidence to prevent UTIs in nursing home residents to inform both bedside care and research efforts. Two types of literature review and summary were performed. First, we conducted a systematic review of individual studies reporting outcomes of UTI, CAUTI, bacteriuria, or urinary catheter use after interventions for reducing catheter use, improving insertion and maintenance of catheters, and/or general infection prevention strategies (eg, improving hand hygiene, infection surveillance, contact precautions, standardizing UTI diagnosis, and antibiotic use). Second, we performed a narrative review to generate an overview of evidence and published recommendations in both acute care and nursing home settings to prevent UTI in catheterized and non-catheterized older adults, which is provided as a comprehensive reference table for clinicians and researchers choosing and refining interventions to reduce UTIs. 


\section{METHODS}

The systematic review was performed according to the criteria of the Preferred Reporting Items for Systematic Reviews and Meta-Analysis recommendations. The protocol was registered at the PROSPERO International Prospective Register of Systematic Reviews, (CRD42013005787). The narrative review was performed using the articles obtained from the systematic search and a targeted literature review by topic for a comprehensive list of interventions, including other interventions summarized in published reviews and guidelines.

\section{Eligibility Criteria Review}

Study Design. To address the breadth and depth of literature available to inform interventions to prevent UTI in nursing homes, broad eligibility criteria were applied with the expectation of varied designs and outcomes. All included studies for the systematic review were published manuscripts reporting a comparison group. We included randomized controlled trials as well as nonrandomized trials (pretest/posttest, with or without concurrent or nonconcurrent controls), with any duration of postintervention follow-up. Observational and retrospective studies were excluded.

Participants. We were interested in interventions and outcomes reported for nursing homes, defined as facilities providing short-stay skilled nursing care and/or rehabilitation, as well as long-term care. We also included evidence derived from rehabilitation facilities and spinal cord injury programs focused on reducing CAUTI risk for chronically catheterized residents. We excluded long-term acute care hospitals, hospice, psychiatric/mental health facilities, pediatric, and community dwelling/outpatient settings.

Interventions. We included interventions involving urinary catheter use such as improving appropriate use, aseptic placement, maintenance care, and prompting removal of unnecessary catheters. We included infection prevention strategies with a particular interest in hand hygiene, barrier precautions, infection control strategies, infection surveillance, use of standardized infection definitions, and interventions to improve antibiotic use. We included single and multiple interventions.

Outcomes

1. Healthcare-associated urinary tract infection: UTI occurring after admission to a healthcare facility, not identified specifically as catheter-associated. We categorized UTI outcomes with as much detail as provided, such as whether the reported outcome included only noncatheter-associated UTIs, the time required after admission (eg, more than 2 days), and whether the UTIs were defined by only laboratory criteria, clinically diagnosed infections, symptomatic, or long-term care specific surveillance definitions.

2. Catheter-associated urinary tract infection: UTI occurring in patients during or immediately after use of a urinary catheter. We noted whether CAUTI was defined by laboratory criteria, clinical symptoms, provider diagnosis, or antimicrobial treatment for case identification. We were primarily interested in CAUTI developing after placing an indwelling urinary catheter, commonly known as a Foley, but also in CAUTI occurring with other catheter types such as intermittent straight catheters, external or "condom" catheters, and suprapubic catheters.

3. Bacteriuria: We included the laboratory-based definition of bacteriuria as an outcome to include studies that reduced asymptomatic bacteriuria.

4. Urinary catheter use measures: This includes measures such as urinary catheter utilization ratios (catheter-days/patient-days), prevalence of urinary catheter use, or percentage of catheters with an appropriate indication.

Study Characteristics for Inclusion. Our systematic search included published papers in the English language. We did not exclude studies based on the number of facilities included or eligible, residents/patients included (based on age, gender, catheter use or type, or antibiotic use), intervention details, study withdrawal, loss to follow-up, death, or duration of pre-intervention and postintervention phases.

\section{Data Sources and Searches}

The following data sources were searched: Ovid MEDLINE (1950 to June 22, 2015), Cochrane Library via Wiley (1960 to June 22, 2015), CINAHL (1981 to June 22, 2015), Web of Science (1926 to June 22, 2015), and Embase.com (1946 to June 22, 2015). Two major systematic search strategies were performed for this review (Figure). Systematic search 1 was designed broadly using all data sources described above to identify interventions aimed at reducing all UTI events (defined under "Outcomes" above) or urinary catheter use (all types), focusing on interventions evaluated in nursing homes. Systematic search 2 was conducted in Ovid MEDLINE to identify studies to reduce UTI events or urinary catheter use measures for patients with a history of longterm or chronic catheter use, including nursing homes and other post-acute care settings such as rehabilitation units or hospitals and spinal cord injury programs, which have large populations of patients with chronic catheter needs. To inform the completeness of the broader systematic searches, supplemental systematic search strategies were performed for specific topics including hydration (supplemental search 1 ), published work by nursing home researchers known to the authors (supplemental search 2), and contact precautions (supplemental search 3). Search 1 is available at http://www.crd.york.ac.uk/PROSPERO/display_record.asp?ID=CRD42013005787. Full search strategies for search 2 and supplemental searches are available upon request.

\section{Study Selection}

One author performed an initial screen of all records retrieved by the systematic searches by title and abstract and applied the initial exclusions (eg, non-human, no outcomes of interest), identified duplicate records, and assigned potentially relevant studies into groups such as review articles, epidemiology, interventions, and articles requiring further text review before categorization (Figure). After initial screening, Dr. Meddings reviewed the records by title/abstract. Reference 


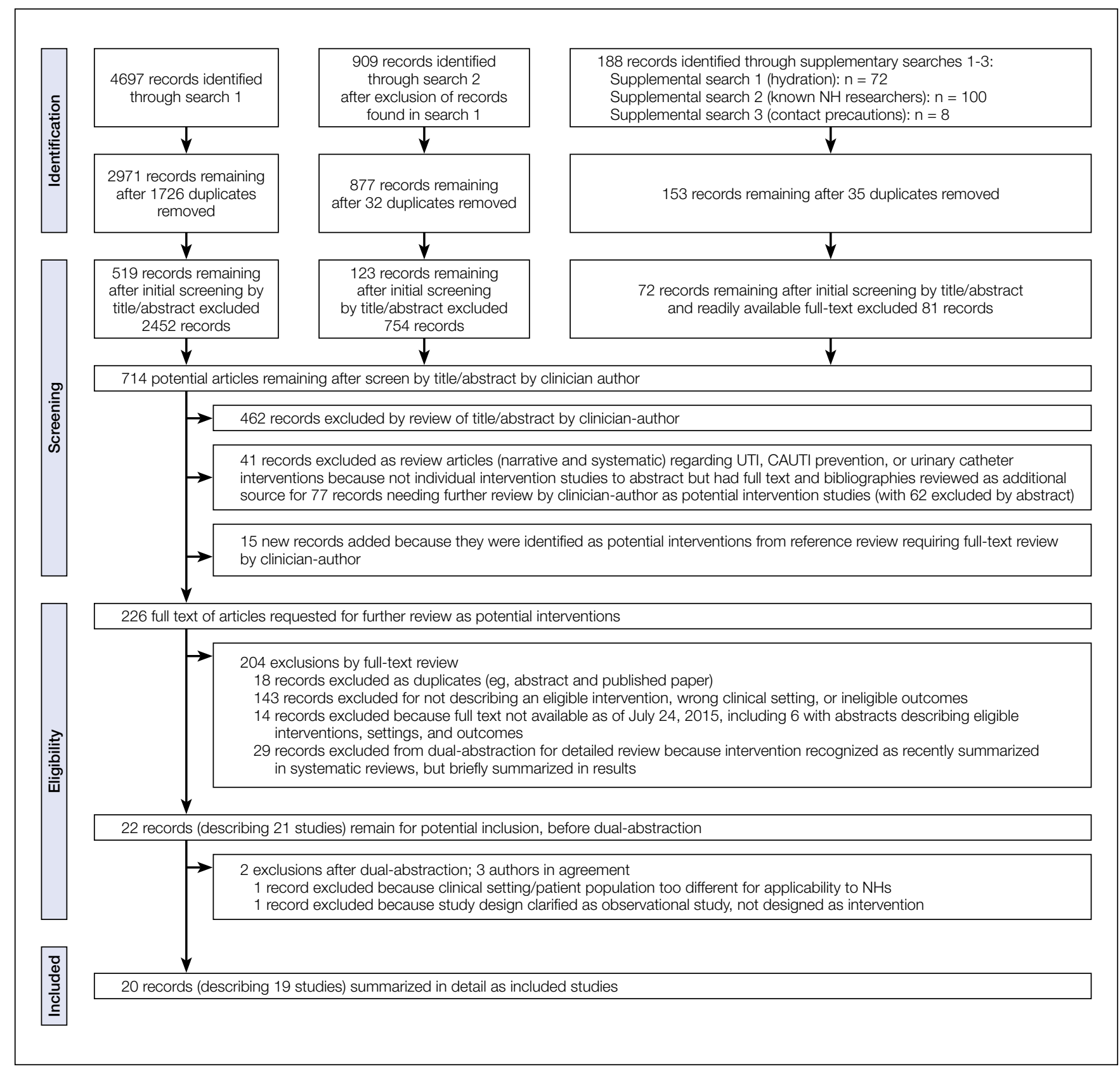

FIG. Study Flow Diagram.

NOTE: Abbreviations: CAUTI, catheter-associated urinary tract infection; NH, nursing home; UTI, urinary tract infection.

lists were reviewed for potential articles for inclusion. Fulltext article review informed the selection of those for dual abstraction and quality scoring performed by 2 authors, with discrepancies resolved by a third author. We requested additional information from authors from whom our search had generated only an abstract or brief report, or when additional information such as pre-intervention data was needed. ${ }^{12-18}$

\section{Data Extraction and Quality Assessment}

Relevant data regarding study design, participants, inclusion/ exclusion criteria, outcomes, and quality criteria were $a b-$ stracted independently by 2 authors. Methodological qual- ity scores were assigned using a modification of the Quality index checklist developed by Downs and Black appropriate for assessing both randomized and nonrandomized studies of healthcare interventions. ${ }^{19}$ We also reviewed study funding sources and other potential quality concerns.

\section{Data Analysis}

Due to large trial heterogeneity among these studies about interventions and outcomes reported, outcome data could not be combined into summary measures for meta-analysis to give overall estimates of treatment effects.

Continued on page 360 
TABLE 1. Characteristics of Included Studies

\begin{tabular}{|c|c|c|c|c|c|c|}
\hline \multirow[b]{2}{*}{ First Author, Year, Country } & \multirow[b]{2}{*}{ Study Design } & \multirow[b]{2}{*}{$\begin{array}{l}\text { Participants/Setting } \\
\text { Total N if provided }\end{array}$} & \multicolumn{3}{|c|}{ Interventions to Reduce UTI, CAUTI or Urinary Catheter Use ${ }^{a}$} & \multirow{2}{*}{$\begin{array}{c}\text { Reported } \\
\text { Outcomes } \\
\text { Types }^{b}\end{array}$} \\
\hline & & & $\begin{array}{l}\text { Strategies to reduce or } \\
\text { improve catheter use }\end{array}$ & $\begin{array}{c}\text { Infection prevention } \\
\text { strategies }\end{array}$ & Other strategies & \\
\hline \multicolumn{7}{|c|}{ Studies including participants who could be with or without urinary catheterization (ie, not limited to catheterized patients only) } \\
\hline Ahlbrecht, 1999, U.S. ${ }^{20}$ & Pre-post NRT & $\begin{array}{l}\text { Residents of a } 220 \text {-bed } \\
\text { community nursing home }\end{array}$ & Maintenance & $\begin{array}{l}\text { Antibiotic review, hand } \\
\text { hygiene, infection control, } \\
\text { standardize UTI diagnosis, } \\
\text { surveillance }\end{array}$ & $\begin{array}{l}\text { Improve resident/patient } \\
\text { hygiene }\end{array}$ & UTI \\
\hline $\begin{array}{l}\text { Brownhill, 2013, United } \\
\text { Kingdom }^{15}\end{array}$ & Pre-post NRT & $\begin{array}{l}\text { Residents of } 47 \text { care } \\
\text { homes }\end{array}$ & $\begin{array}{l}\text { Maintenance, catheter se- } \\
\text { curement, standard supplies, } \\
\text { incontinence care, other: } \\
\text { more leg/night bag sizes, } \\
\text { improve urine sampling }\end{array}$ & $\begin{array}{l}\text { Antibiotic use review, } \\
\text { standard UTI diagnosis } \\
\text { definitions }\end{array}$ & $\begin{array}{l}\text { Programs to reduce falls and } \\
\text { pressure ulcers }\end{array}$ & UTI, CAUTI \\
\hline Cools, 1988, The Netherlands ${ }^{21}$ & Pre-post NRT & $\begin{array}{l}320 \text {-bed skilled nursing } \\
\text { facility }\end{array}$ & $\begin{array}{l}\text { Appropriate indications, } \\
\text { prompt removal, inconti- } \\
\text { nence care }\end{array}$ & $\begin{array}{l}\text { Antibiotic guide, hand } \\
\text { hygiene, infection control, } \\
\text { standardize UTI definitions, } \\
\text { surveillance. }\end{array}$ & $\begin{array}{l}\text { Weekly data and new } \\
\text { patient review by physicians; } \\
\text { improve ventilation by chairs, } \\
\text { exercise, physiotherapy }\end{array}$ & UTI, catheter use \\
\hline Fendler, 2002, U.S. ${ }^{22}$ & $\begin{array}{l}\text { NRT with concurrent } \\
\text { internal and external } \\
\text { controls }\end{array}$ & $\begin{array}{l}\text { Residents of a } 275 \text {-bed } \\
\text { extended care facility } \\
\text { providing rehabilitation } \\
\text { and subacute care }\end{array}$ & None specified & Hand hygiene & None specified & CAUTI \\
\hline Klay, 2005, U.S. ${ }^{23}$ & $\begin{array}{l}\text { Pre-post NRT of same } \\
\text { patients }\end{array}$ & $\begin{array}{l}42 \text { female residents with } \\
\text { urinary incontinence in } \\
1 \text { extended care facility }\end{array}$ & Incontinence care & None specified & $\begin{array}{l}\text { Family education on } \\
\text { incontinence }\end{array}$ & UTI \\
\hline Lin, 2013, Taiwan²4 & $\begin{array}{l}\text { Pre-post NRT with } \\
\text { external controls }\end{array}$ & $\begin{array}{l}\text { Incontinent residents of } \\
6 \text { nursing homes }\end{array}$ & None specified & None specified & Increase hydration & Bacteriuria \\
\hline \multirow[t]{2}{*}{ McConnell, 1984, U.S. ${ }^{25}$} & Pre-post NRT & $\begin{array}{l}102 \text { residents of nursing } \\
\text { home }\end{array}$ & $\begin{array}{l}\text { Appropriate indications, } \\
\text { prompt removal }\end{array}$ & None specified & $\begin{array}{l}\text { Increase hydration, ambula- } \\
\text { tion program }\end{array}$ & UTI \\
\hline & & & Incontinence care & & & \\
\hline Mentes, 2003, U.S. ${ }^{26}$ & $\begin{array}{l}\text { RCT-cluster (random- } \\
\text { ized at facility level) }\end{array}$ & $\begin{array}{l}42 \text { elderly residents from } \\
4 \text { nursing homes ( } 2 \text { VA } \\
\text { nursing homes, } 2 \text { com- } \\
\text { munity nursing homes) }\end{array}$ & None specified & None specified & Increase hydration & UTI \\
\hline Miller, 2014, U.S. ${ }^{27}$ & $\begin{array}{l}\text { Pre-post NRT panel } \\
\text { survey of stratified } \\
\text { proportionate random } \\
\text { sample of nursing } \\
\text { directors and admin- } \\
\text { istrators compared to } \\
\text { resident outcomes }\end{array}$ & $\begin{array}{l}824 \text { nursing homes in } \\
\text { large study on implemen- } \\
\text { tation of culture change } \\
\text { between } 2005-2010\end{array}$ & $\begin{array}{l}\text { Comprehensive "culture } \\
\text { change program" anticipated } \\
\text { to improve measures includ- } \\
\text { ing percentage on bladder } \\
\text { training programs and } \\
\text { reduction of UTI events }\end{array}$ & None specified & $\begin{array}{l}\text { Introduction of "culture } \\
\text { change practices," as quan- } \\
\text { tified by a culture change } \\
\text { practice score reflecting } \\
3 \text { domains: nursing home } \\
\text { environment, resident-cen- } \\
\text { tered care involving bladder } \\
\text { training programs, staff } \\
\text { empowerment }\end{array}$ & UTI \\
\hline Stuart, 2015, Australia ${ }^{28}$ & Pre-post NRT & $\begin{array}{l}\text { Residents in } 2 \text { urban aged } \\
\text { care facilities; } 130 \text { beds }\end{array}$ & None specified & $\begin{array}{l}\text { Nurse-led antibiotic stew- } \\
\text { ardship program, infection } \\
\text { control, and surveillance } \\
\text { programs }\end{array}$ & $\begin{array}{l}\text { Nurse-physician commu- } \\
\text { nications about antibiotics } \\
\text { and data }\end{array}$ & UTI \\
\hline $\begin{array}{l}\text { Van Gaal, 2011, The Nether- } \\
\text { lands }{ }^{29,30}\end{array}$ & $\begin{array}{l}\text { RCT-cluster (random- } \\
\text { ized at ward level) }\end{array}$ & $\begin{array}{l}392 \text { residents from } 10 \\
\text { wards in } 6 \text { nursing homes }\end{array}$ & $\begin{array}{l}\text { Hand hygiene/gloves for } \\
\text { catheter/bag contact, } \\
\text { appropriate indications, } \\
\text { standard catheter supplies, } \\
\text { maintenance, catheter se- } \\
\text { curement, prompt removal, } \\
\text { incontinence care }\end{array}$ & Surveillance & $\begin{array}{l}\text { Fall, pressure ulcer, UTI } \\
\text { prevention programs with } \\
\text { nurse education/feedback }\end{array}$ & $\begin{array}{l}\text { UTI } \\
\text { Catheter use }\end{array}$ \\
\hline Yeung, 2011, China ${ }^{31}$ & $\begin{array}{l}\text { RCT-cluster (random- } \\
\text { ized at facility level), } \\
\text { unblended }\end{array}$ & $\begin{array}{l}1268 \text { elderly residents in } \\
6 \text { nursing homes }\end{array}$ & None specified & Hand hygiene & None specified & UTI \\
\hline \multicolumn{7}{|c|}{ Studies including only catheterized participants or in settings where very high urinary catheterization rates expected } \\
\hline Darouiche, 2006, U.S. ${ }^{32}$ & $\begin{array}{l}\text { RCT } \\
\text { single-blind }\end{array}$ & $\begin{array}{l}127 \text { adults with spinal } \\
\text { cord injury with long-term } \\
\text { indwelling catheters, } 4 \\
\text { hospitals }\end{array}$ & $\begin{array}{l}\text { Catheter securement by } \\
\text { StatLock device (C.R. Bard, } \\
\text { Inc., Covington, Georgia) }\end{array}$ & None specified & None specified & CAUTI \\
\hline Evans, 2013, U.S. ${ }^{33}$ & Pre-post NRT & $\begin{array}{l}22 \text { VA acute care spinal } \\
\text { cord injury units }\end{array}$ & None specified & $\begin{array}{l}\text { MRSA bundle of surveillance, } \\
\text { contact precautions, hand } \\
\text { hygiene }\end{array}$ & Institutional culture change & UTI \\
\hline
\end{tabular}


TABLE 1. Characteristics of Included Studies (continued)

\begin{tabular}{|c|c|c|c|c|c|c|}
\hline \multirow[b]{2}{*}{ First Author, Year, Country } & \multirow[b]{2}{*}{ Study Design } & \multirow[b]{2}{*}{$\begin{array}{l}\text { Participants/Setting } \\
\text { Total } \mathrm{N} \text { if provided }\end{array}$} & \multicolumn{3}{|c|}{ Interventions to Reduce UTI, CAUTI or Urinary Catheter Use ${ }^{a}$} & \multirow{2}{*}{$\begin{array}{c}\text { Reported } \\
\text { Outcomes } \\
\text { Types }^{\text {b }}\end{array}$} \\
\hline & & & $\begin{array}{l}\text { Strategies to reduce or } \\
\text { improve catheter use }\end{array}$ & $\begin{array}{l}\text { Infection prevention } \\
\text { strategies }\end{array}$ & Other strategies & \\
\hline Mody, 2015, U.S. ${ }^{34}$ & $\begin{array}{l}\text { RCT-cluster (random- } \\
\text { ized at facility level) }\end{array}$ & $\begin{array}{l}418 \text { residents with devic- } \\
\text { es (catheters or feeding } \\
\text { tubes) in } 12 \text { community } \\
\text { nursing homes }\end{array}$ & $\begin{array}{l}\text { Hand hygiene promotion } \\
\text { including gown/gloves when } \\
\text { working with indwelling } \\
\text { devices }\end{array}$ & $\begin{array}{l}\text { Standardize UTI diagnosis, } \\
\text { hand hygiene/gown/gloves } \\
\text { with morning/evening patient } \\
\text { care, splashing activity, } \\
\text { MDRO-active surveillance, } \\
\text { pre-emptive barrier precau- } \\
\text { tions if device }\end{array}$ & Staff program education & CAUTI \\
\hline Priefer, 1982, U.S. ${ }^{35}$ & RCT & $\begin{array}{l}17 \text { male residents with } \\
\text { indwelling catheters in } 1 \\
\text { VA nursing home }\end{array}$ & $\begin{array}{l}\text { Scheduled catheter change } \\
\text { (monthly + for block/infec- } \\
\text { tion) compared to change } \\
\text { only for block/infection }\end{array}$ & None specified & None specified & CAUTI \\
\hline Saint, 2006, U.S. ${ }^{36}$ & $\begin{array}{l}\text { RCT } \\
\text { unblinded }\end{array}$ & $\begin{array}{l}75 \text { men }>40 \text { years } \\
\text { requiring a urinary } \\
\text { collection device in } 1 \text { VA } \\
\text { hospital's units (medicine, } \\
\text { neuro, rehab, nursing } \\
\text { home) }\end{array}$ & $\begin{array}{l}\text { Condom catheterization vs. } \\
\text { indwelling Foley catheter- } \\
\text { ization }\end{array}$ & None specified & None specified & $\begin{array}{l}\text { Bacteriuria, and } \\
\text { composite of } \\
\text { bacteriuria or } \\
\text { CAUTI or death }\end{array}$ \\
\hline Suardi, 2001, Italy ${ }^{37}$ & $\begin{array}{l}\text { Pre-post NRT, for } \\
\text { same patients }\end{array}$ & $\begin{array}{l}20 \text { spinal cord injury } \\
\text { rehab patients with neu- } \\
\text { rogenic bladder with inter- } \\
\text { mittent catheterization }\end{array}$ & $\begin{array}{l}\text { Time-volume dependent } \\
\text { catheterization using bladder } \\
\text { scanner }\end{array}$ & None specified & None specified & Catheter use \\
\hline Tang, 2006, China ${ }^{38}$ & RCT & $\begin{array}{l}81 \text { females with urinary } \\
\text { retention in geriatric } \\
\text { rehab ward }\end{array}$ & $\begin{array}{l}\text { Comparing intermittent vs. } \\
\text { indwelling catheters, bladder } \\
\text { scan protocol }\end{array}$ & None specified & None specified & $\begin{array}{l}\text { CAUTI, } \\
\text { bacteriuria }\end{array}$ \\
\hline
\end{tabular}

asupplemental Table 2 provides details of the interventions, duration of study, and measure collection details.

bUTI: urinary tract infection not identified specifically as catheter-associated; bacteriuria: bacteriuria, not otherwise identified as UTI or CAUTl; outcome results provided in Table 2 .

NOTE: Abbreviations: CAUTI: catheter-associated urinary tract infection; MDRO, multidrug resistant organism; MRSA, methicillin-resistant Staphylococcus aureus; NRT, nonrandomized trial; RCT, randomized controlled trial; VA, Veterans Affairs.

\section{RESULTS}

\section{Systematic Search Results and Study Selection}

As detailed in the study flow diagram (Figure), 5794 total records were retrieved by systematic search 1 (4697 studies), search 2 (909 studies), and supplemental searches (188 studies). Hand searching of reference lists of 41 reviews (including narrative and systematic reviews) yielded 77 additional studies for consideration. Twenty-nine records on interventions that were the focus of systematic reviews, including topics of cranberry use, catheter coatings, antimicrobial prophylaxis, washout/irrigation strategies, and sterile versus clean intermittent straight catheterization, were excluded from dual abstraction. Two records were excluded after team discussion of the dual-abstraction results, because 1 study did not meet criteria as an intervention study and 1 study's setting was not applicable in nursing homes. A total of 20 records $^{15,20-38}$ (in which 19 studies were described) were selected for final inclusion for detailed assessment and reporting for the systematic review.

\section{Characteristics of Included Studies}

Table 1 describes the 19 intervention studies in terms of design, participants, setting, and whether the study included specific categories of interventions expected to decrease UTI or catheter use. These studies included 8 randomized controlled trials ( 4 with cluster-randomization at the facility or unit level), 10 pre-post nonrandomized interventions, and 1 nonrandomized intervention with concurrent con- trols. Twelve studies included participants with or without catheters (ie, not limited to catheterized patients only) in nursing homes. ${ }^{15,20-31}$ Seven ${ }^{32-38}$ studies included catheterized patients only or settings with high expected catheterization rates; settings for these studies included spinal cord units $(n=3)$, nursing homes $(n=2)$, rehabilitation ward $(n=1)$ and VA hospital $(\mathrm{n}=1)$, including acute care, nursing home, and rehabilitation units. Total quality scores for the studies ranged from 8 to 25 (median, 15), detailed in Supplemental Table 1.

As detailed in Table 1 and Supplemental Table 2, 7 studies $^{22,24,26,31,32,35,36}$ involved single interventions and 12 studies ${ }^{15,20,21,23,25,27-30,33,34,37,38}$ included multiple interventions. Interventions to impact catheter use and care were evaluated in 13 studies, including appropriateness of use,,$^{21,25,29,30}$ improving catheter maintenance care, ${ }^{15,20,29,30}$ securement, ${ }^{15,29,30,32}$ prompting removal of unnecessary catheters, ${ }^{21,25,29,30}$ improving incontinence care, ${ }^{15,21,23,25}$ bladder scanners, ${ }^{37,38}$ catheter changes, ${ }^{35}$ and comparing alternatives (condom catheter or intermittent straight catheter) to use of an indwelling catheter. ${ }^{36,38}$ None focused on improving aseptic insertion. General infection control practices studied included improving hand hygiene, ${ }^{20-22,29-31,33,34}$ improving antibiotic use, ${ }^{15,20,21,28,34}$ initiation of infection control programs, ${ }^{20,21,28}$ interventions to improve identification of UTIs/CAUTIs using infection symptom/sign criteria, $15,20,21,34$ infection surveillance as an intervention, ${ }^{28-30,33,34}$ and barrier precautions, ${ }^{33,34}$ including

Continued on page 365 
TABLE 2. Summary of Outcomes from Included Studies

\begin{tabular}{|c|c|c|c|c|c|c|}
\hline First Author, Year & $\begin{array}{l}\text { UTI, CAUTI, } \\
\text { Bacteriuria measures }\end{array}$ & Comparison Group & Intervention & $\begin{array}{l}\text { Urinary Catheter } \\
\text { Use Measures }\end{array}$ & Comparison Group & Intervention \\
\hline \multicolumn{7}{|c|}{ Studies including participants who could be with or without urinary catheterization (ie, not limited to catheterized patients only) } \\
\hline \multirow[t]{2}{*}{ Ahlbrecht, $1999^{20}$} & $\begin{array}{l}\text { Overall UTI rate/1000 } \\
\text { resident days }\end{array}$ & 1.18 (Cl: 0.36, 2.01) & $\begin{array}{l}1.14 \text { (Cl: } 0.94,1.34), P= \\
0.65\end{array}$ & None reported & & \\
\hline & $\begin{array}{l}\text { UTIs in nonambulatory } \\
\text { females without indwelling } \\
\text { catheters/1000 resident } \\
\text { days }\end{array}$ & 2.40 (Cl: $1.96,2.84)$ & $\begin{array}{l}3.06 \text { (Cl: } 2.19,3.93), P= \\
0.05\end{array}$ & Not applicable & & \\
\hline \multirow[t]{2}{*}{ Brownhilla $2013^{15}$} & Mean UTI/month & 55 UTIS & 18.8 UTIs & Not reported & & \\
\hline & Mean CAUTI/month & 18.3 CAUTIS & 4.3 CAUTIS & Not reported & & \\
\hline Cools, $1988^{21}$ & $\begin{array}{l}\text { UTIs treated with antimi- } \\
\text { crobials (includes with and } \\
\text { without catheters) }\end{array}$ & $\begin{array}{l}0.49 \text { ( } 256 \text { UTIs in } 515 \\
\text { residents) in } \\
\text { year } 1\end{array}$ & $\begin{array}{l}0.125 \text { (66 UTIs in } 527 \\
\text { residents in year } 6\end{array}$ & $\begin{array}{l}\text { Prevalence (\%) } \\
\text { of indwelling } \\
\text { catheters }\end{array}$ & Year $1=21 \%(109 / 515)$ & Year $6=10 \%(52 / 527)$ \\
\hline Fendler, $2002^{22}$ & $\begin{array}{l}\text { CAUTIs per } 1000 \text { pa- } \\
\text { tient-days, by symptomatic } \\
\text { infection, } 1991 \text { McGeer } \\
\text { criteria }^{42}\end{array}$ & $\begin{array}{l}0.77 \text { (133 CAUTIs per } \\
172,897 \text { patient-days) }\end{array}$ & $\begin{array}{l}0.63 \text { (51 CAUTIs per 81,036 } \\
\text { patient-days) }\end{array}$ & Not reported & & \\
\hline Klay, $2005^{23}$ & $\begin{array}{l}\text { Number of UTIs (not de- } \\
\text { fined further by symptom } \\
\text { or catheter-association) }\end{array}$ & 31 UTIS & 6 UTIs & Not reported & & \\
\hline \multirow[t]{7}{*}{ Lin, $2013^{24}$} & \multirow{7}{*}{$\begin{array}{l}\text { Asymptomatic bacteriuria } \\
\text { in patients without indwell- } \\
\text { ing catheters }\end{array}$} & Control group & Intervention group & \multirow[t]{7}{*}{ Not reported } & & \\
\hline & & Baseline: & Baseline: & & & \\
\hline & & $16.7 \%(n=5$ of 30$)$ & $38.6 \%(n=17$ of 44$)$ & & & \\
\hline & & Follow-up: & & & & \\
\hline & & $10 \%(n=3$ of 30$)$ & Post-intervention: & & & \\
\hline & & & $22.7 \%(n=10$ of 44$)$ & & & \\
\hline & & \multicolumn{2}{|c|}{ No significant bacteriuria for either group } & & & \\
\hline McConnell, $1984^{25}$ & $\begin{array}{l}\text { Number of UTIs (unclear if } \\
\text { restricted to symptomatic; } \\
\text { population seems to } \\
\text { include both those with } \\
\text { and without catheters) }\end{array}$ & $\begin{array}{l}\text { Monthly rates of 3-9 UTIs } \\
\text { in months June-November } \\
1982 \text { (before full imple- } \\
\text { mentation in December } \\
\text { 1982) }\end{array}$ & $\begin{array}{l}\text { Monthly rates of } 1-3 \text { UTI } \\
\text { in December-June } 1982 \\
\text { (after December } 1982 \text { full } \\
\text { implementation) }\end{array}$ & Not reported & & \\
\hline Mentes, $2003^{26}$ & $\begin{array}{l}\text { Hydration-linked event } \\
\text { of UTI diagnosed by } \\
\text { a provider (unclear if } \\
\text { symptoms, catheter use, or } \\
\text { other criteria), proceeded } \\
\text { by urine specific gravity of } \\
\geq 1.010 \text { and decreased } \\
\text { fluid intake }\end{array}$ & $\begin{array}{l}1 \text { UTI ( } 4.1 \% \text { of } 24 \text { control } \\
\text { patients) }\end{array}$ & $\begin{array}{l}0 \text { UTI ( } 0 \% \text { of } 25 \text { treatment } \\
\text { patients) }\end{array}$ & Not reported & & \\
\hline \multirow[t]{4}{*}{ Miller, 2014²7 } & \multirow{4}{*}{$\begin{array}{l}\text { Percentage of residents } \\
\text { with UTI in last } 30 \text { days } \\
\text { reported in Minimum } \\
\text { Data Set: }\end{array}$} & $\begin{array}{l}531 \mathrm{NHs} \text { in bottom } 3 \\
\text { quartiles of culture change } \\
\text { composite score }\end{array}$ & $\begin{array}{l}207 \mathrm{NHs} \text { in top quartile of } \\
\text { culture change composite } \\
\text { score }\end{array}$ & \multirow[t]{4}{*}{ Not reported } & & \\
\hline & & $\begin{array}{l}\text { Baseline period: } \\
8.4 \% \pm 5.6 \text { (SD) }\end{array}$ & $\begin{array}{l}\text { Baseline period: } \\
8.8 \% \pm 4.9 \text { (SD) }\end{array}$ & & & \\
\hline & & $\begin{array}{l}\text { Follow-up period: } \\
8.9 \% \pm 5.4 \text { (SD) }\end{array}$ & $\begin{array}{l}\text { Follow-up period: } \\
8.6 \% \pm 5.1 \text { (SD) } \\
\end{array}$ & & & \\
\hline & & $\begin{array}{l}\text { Coefficient }+0.72 \text { (SE, } \\
0.28), \text { meaning higher UTI } \\
\text { rates, } P=0.01\end{array}$ & $\begin{array}{l}\text { Coefficient }-0.06 \text { (SE, 0.54), } \\
P=0.92\end{array}$ & & & \\
\hline Stuart, $2015^{28}$ & $\begin{array}{l}\text { UTI rates form surveillance } \\
\text { data using McGeer's } \\
\text { criteria }\end{array}$ & $\begin{array}{l}\text { Data not provided, but text ir } \\
\text { rates surveillance data rema } \\
\text { collection periods }\end{array}$ & $\begin{array}{l}\text { dicates surveillance infection } \\
\text { ined stable over the } 2 \text { data }\end{array}$ & Not reported & & \\
\hline \multirow{5}{*}{$\begin{array}{l}\text { Van Gaal, } \\
2011^{29,30}\end{array}$} & \multirow{5}{*}{$\begin{array}{l}\text { Symptomatic UTI } \\
\text { confirmed by physician, } \\
\text { reported as incidence rate } \\
\text { per patient per week }\end{array}$} & Baseline period: & Baseline period: & \multirow{5}{*}{$\begin{array}{l}\text { Patients with indwelling } \\
\text { catheters with a correct } \\
\text { indication (\%) }\end{array}$} & \multirow{5}{*}{$\begin{array}{l}\text { Usual care } \\
\text { Baseline: } 6 \% \\
\text { Follow-up: } 34 \%\end{array}$} & \multirow{5}{*}{$\begin{array}{l}\text { Intervention } \\
\text { Baseline: 34\% } \\
\text { Follow-up: 32\% }\end{array}$} \\
\hline & & $\mathrm{n}=28$ UTIs for $127 \mathrm{pa}-$ & $\mathrm{n}=23$ UTIs for 114 patients, & & & \\
\hline & & $\begin{array}{l}\text { tients, occurring at rate of } \\
0.03 \text { per patient per week }\end{array}$ & $\begin{array}{l}\text { occurring at rate of } 0.03 \text { per } \\
\text { patient per week }\end{array}$ & & & \\
\hline & & $\begin{array}{l}\text { Follow-up period: } \\
\mathrm{n}=57 \text { UTIs for } 196 \mathrm{pa-} \\
\text { tients, occurring at rate of } \\
0.02 \text { per patient per week }\end{array}$ & $\begin{array}{l}\text { Follow-up period: } \\
\mathrm{n}=58 \text { UTls for } 196 \text { patients, } \\
\text { occurring at rate of } 0.02 \text { per } \\
\text { patient per week }\end{array}$ & & & \\
\hline & & \multicolumn{2}{|c|}{$\begin{array}{l}\text { Overall UTI outcome for this study, reported as ratio of UTIs } \\
\text { in intervention versus usual care group: } 0.85 \text { with } 96 \% \mathrm{Cl} \text { : } \\
0.43-1.67\end{array}$} & & & \\
\hline
\end{tabular}


TABLE 2. Summary of Outcomes from Included Studies (continued)

\begin{tabular}{|c|c|c|c|c|c|c|}
\hline First Author, Year & $\begin{array}{l}\text { UTI, CAUTI, } \\
\text { Bacteriuria measures }\end{array}$ & Comparison Group & Intervention & $\begin{array}{l}\text { Urinary Catheter } \\
\text { Use Measures }\end{array}$ & Comparison Group & Intervention \\
\hline \multirow[t]{3}{*}{ Yeung, $2011^{31}$} & \multirow[t]{3}{*}{$\begin{array}{l}\text { UTIs requiring hospital- } \\
\text { ization, unclear if with or } \\
\text { without catheters }\end{array}$} & $\begin{array}{l}\text { Baseline period: } \\
3 \text { UTIs per 32,726 resi- } \\
\text { dent-days, calculated as }\end{array}$ & $\begin{array}{l}\text { Baseline period: } \\
6 \text { UTIs per } 21,862 \text { resi- } \\
\text { dent-days, calculated as } 0.27\end{array}$ & \multirow[t]{3}{*}{ Not reported } & & \\
\hline & & $\begin{array}{l}0.09 \text { per } 1000 \text { resi- } \\
\text { dent-days }\end{array}$ & $\begin{array}{l}\text { per } 1000 \text { resident-days } \\
\text { Follow-up period: }\end{array}$ & & & \\
\hline & & $\begin{array}{l}\text { Follow-up period: } \\
22 \text { UTIs per } 81,177 \\
\text { resident-days, calculated } \\
\text { as } 0.27 \text { per } 1000 \text { resi- } \\
\text { dent-days, } P=0.06\end{array}$ & $\begin{array}{l}8 \text { UTIs per } 50,441 \text { resi- } \\
\text { dent-days, calculated as } 0.16 \\
\text { per } 1000 \text { resident-days), } P \\
=0.30\end{array}$ & & & \\
\hline \multicolumn{7}{|c|}{ Studies including only catheterized participants or in settings where very high urinary catheterization rates expected } \\
\hline \multirow[t]{3}{*}{$\begin{array}{l}\text { Darouiche, } \\
2006^{32}\end{array}$} & $\begin{array}{l}\text { Number of symptomatic } \\
\text { CAUTIs in patients with } \\
\text { Foley or suprapubic } \\
\text { catheters }\end{array}$ & $\begin{array}{l}14 \text { CAUTIs ( } 24.1 \% \text { of } 58 \\
\text { patients followed) }\end{array}$ & $\begin{array}{l}8 \text { CAUTIs ( } 13.3 \% \text { of } 60 \\
\text { patients followed). RR=0.55, } \\
95 \% \text { Cl: } 0.25-1.22 ; P=0.16\end{array}$ & Not reported & & \\
\hline & \multirow{2}{*}{$\begin{array}{l}\text { Symptomatic CAUTI rate } \\
\text { as CAUTIs per } 1000 \text { device } \\
\text { days }\end{array}$} & \multirow{2}{*}{$\begin{array}{l}\text { 4.9 CAUTI per } 1000 \text { device } \\
\text { days }\end{array}$} & $\begin{array}{l}2.7 \text { CAUTI per } 1000 \text { device } \\
\text { days, } P=0.16\end{array}$ & Not reported & & \\
\hline & & & $\begin{array}{l}\text { but study not powered to } \\
\text { detect significant change }\end{array}$ & & & \\
\hline Evans, $2013^{33}$ & $\begin{array}{l}\text { MRSA hospital-associated } \\
\text { UTIs }\end{array}$ & $\begin{array}{l}\text { Actual Ns and rates were } \\
\text { not provided in report }\end{array}$ & $\begin{array}{l}\text { Quarterly UTI rates declined by } \\
33 \%(P=0.07)\end{array}$ & Not reported & & \\
\hline \multirow[t]{2}{*}{ Mody, $2015^{34}$} & $\begin{array}{l}\text { Clinically-defined (symp- } \\
\text { tomatic) first new CAUTIs } \\
\text { per } 1000 \text { device-days }\end{array}$ & $\begin{array}{l}10.0 \text { CAUTIs per } 1000 \\
\text { device-days }\end{array}$ & $\begin{array}{l}5.2 \text { CAUTIs per } 1000 \text { de- } \\
\text { vice-days (HR, } 0.54 \text {; }(95 \% \mathrm{Cl} \text { : } \\
0.30,0.97), P=0.04^{\mathrm{b}}\end{array}$ & Not reported & & \\
\hline & $\begin{array}{l}\text { Clinically-defined (symp- } \\
\text { tomatic) all new CAUTIs } \\
\text { (includes recurrent) per } \\
1000 \text { device-days }\end{array}$ & $\begin{array}{l}\text { 9.2 CAUTIs per } 1000 \\
\text { device-days }\end{array}$ & $\begin{array}{l}5.9 \text { CAUTIs per } 1000 \\
\text { device-days } \\
\text { (HR, } 0.69 \text { ( } 95 \% \text { Cl: } 0.49 \text {, } \\
0.99), P=0.045^{\mathrm{b}}\end{array}$ & Not reported & & \\
\hline \multirow[t]{2}{*}{ Priefer, $1982^{35}$} & $\begin{array}{l}\text { Number (\%) of patients } \\
\text { with symptomatic CAUTI } \\
\text { in patients with indwelling } \\
\text { catheters }\end{array}$ & $\begin{array}{l}\text { Control group: } \\
6 \text { of } 7(83 \%) \text { men }\end{array}$ & $\begin{array}{l}\text { Experimental } \\
3 \text { of } 10(30 \%) \text { men }\end{array}$ & Not reported & & \\
\hline & $\begin{array}{l}\text { Number of symptomatic } \\
\text { CAUTIs per patient in } \\
6 \text { months in indwelling } \\
\text { catheter patients }\end{array}$ & $\begin{array}{l}\text { Control group: } \\
1.0 \pm 0.6\end{array}$ & $\begin{array}{l}\text { Experimental: } \\
0.4 \pm 0.7 \\
P>0.05\end{array}$ & Not reported & & \\
\hline \multirow[t]{5}{*}{ Saint, $2006^{36}$} & $\begin{array}{l}\text { Number with bacteriuria } \\
\left(\geq 10^{3} \mathrm{CFUs} \mathrm{per} \mathrm{mL} \text { of sin- }\right. \\
\text { gle/predominant species) }\end{array}$ & $\begin{array}{l}\text { Indwelling catheters: } \\
\mathrm{n}=17 \text { (SE, 41.5) }\end{array}$ & $\begin{array}{l}\text { Condom catheter group: } \\
n=13(S E, 38.2)\end{array}$ & Not reported & & \\
\hline & $\begin{array}{l}\text { Bacteriuria per } 1000 \\
\text { patient-days }(95 \% \mathrm{Cl})\end{array}$ & $\begin{array}{l}\text { Indwelling catheters: } \\
\text { 111/1000 patient-days, } \\
95 \% \text { Cl (69-178) }\end{array}$ & $\begin{array}{l}\text { Condom catheter group: } \\
\text { 61/1000 patient-days with } \\
95 \% \mathrm{Cl}(35-104), P=0.11\end{array}$ & Not reported & & \\
\hline & \multirow{2}{*}{$\begin{array}{l}\text { Composite outcome: } \\
\text { number with bacteriuria } \\
\text { or CAUTI (defined by bac- } \\
\text { teriuria and } \geq 1 \text { UTI sign/ } \\
\text { symptom) or death }\end{array}$} & $\begin{array}{l}\text { Indwelling catheters: } \\
\mathrm{n}=20(48.8 \%)\end{array}$ & $\begin{array}{l}\text { Condom catheter group: } \\
\mathrm{n}=15(44.1 \%)\end{array}$ & \multirow[t]{2}{*}{ Not reported } & & \\
\hline & & \multicolumn{2}{|c|}{$\begin{array}{l}\mathrm{HR}, 2.11(95 \% \mathrm{Cl}, 1.03-4.31), P=0.04 \text { comparing this } \\
\text { event in those with indwelling vs. condom catheters }\end{array}$} & & & \\
\hline & $\begin{array}{l}\text { Composite outcome: com- } \\
\text { bined event (bacteriuria or } \\
\text { CAUTI or death) per } 1000 \\
\text { patient-days }\end{array}$ & $\begin{array}{l}\text { Indwelling catheters: } \\
131 \text { per } 1000 \text { patient-days } \\
\text { with } 95 \% \mathrm{Cl}(85-203)\end{array}$ & $\begin{array}{l}\text { Condom catheter group: } \\
70 \text { per } 1,000 \text { patient-days } \\
\text { with } 95 \% \mathrm{Cl}(42-116) \text {, } \\
P=0.07\end{array}$ & Not reported & & \\
\hline Suardi, $2001^{37}$ & Not reported & & & $\begin{array}{l}\text { Number of intermittent } \\
\text { catheterizations and } \\
\text { indwelling catheters } \\
\text { used }\end{array}$ & No Ns reported & $\begin{array}{l}\text { No Ns reported. By } \\
\text { text, reduced indwelling } \\
\text { catheters, } P<0.001^{\mathrm{b}}\end{array}$ \\
\hline \multirow[t]{2}{*}{ Tang, $2006^{38}$} & $\begin{array}{l}\text { Symptomatic CAUTI by } \\
\text { day } 14\end{array}$ & $\begin{array}{l}\text { Indwelling catheter } \\
\text { group: } 0\end{array}$ & $\begin{array}{l}\text { Intermittent catheter group: } 1 \text {. } \\
P=0.400\end{array}$ & $\begin{array}{l}\text { Days to become } \\
\text { catheter-free }\end{array}$ & $\begin{array}{l}\text { Indwelling catheters: } \\
9.2 \pm 4.0 \text { days }\end{array}$ & $\begin{array}{l}\text { Intermittent catheters: } \\
8.6 \pm 3.3 \text { days } P=0.609\end{array}$ \\
\hline & Bacteriuria by day 14 & $\begin{array}{l}\text { Indwelling catheter group: } \\
21 \text { of } 34(61.8 \%) P=0.888\end{array}$ & $\begin{array}{l}\text { Intermittent catheter group: } \\
14 \text { of } 22(63.6 \%)\end{array}$ & $\begin{array}{l}\text { Number patients } \\
\text { catheter-free by day } 14 \\
\text { with postvoid residual } \\
<150 \mathrm{~mL}\end{array}$ & $\begin{array}{l}\text { Indwelling catheters: } \\
27 \text { of } 39(69.2 \%) \text {, }\end{array}$ & $\begin{array}{l}\text { Intermittent catheters: } \\
16 \text { of } 27(59.3 \%) \\
P=0.403\end{array}$ \\
\hline
\end{tabular}

a Study author provided outcome data not in published article.

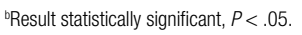

NOTE: Abbreviations: CAUTI: catheter-associated urinary tract infection; CFU, colony-forming units; Cl, 95\% confidence intervals; UTI, urinary tract infection not specified as catheter-associated; HR, hazard ratio; MRSA, methicillin-resistant Staphylococcus aureus; NH, nursing home; SD, standard deviation. SE, standard error. 


\section{TABLE 3. Comprehensive List of Interventions Considered for Prevention of UTI and CAUTI}

This table includes a comprehensive list of potential interventions that have been considered for prevention of UTI or CAUTI (including those in acute and long-term settings), as summarized from this evidence report, and prior comprehensive narrative ${ }^{43-57}$ or systematic reviews. ${ }^{11,58-68}$ Blue-shaded cells describe interventions that are not recommended based on available evidence or rationale. Nonshaded cells describe interventions that have some evidence of benefit (not always from controlled-intervention studies) for certain populations and settings.

Interventions General Summary of Available Evidence and Recommendations Provided

Interventions for Patients Regardless of Urinary Catheter Status

Hand hygiene Interventions to improve hand hygiene have been studied as single interventions ${ }^{22,31}$ and part of bundles ${ }^{12,21,3,3,34}$ for prevention of UTI and CAUTl in LTC settings with decreased (without statistical significance) CAUTI rates ${ }^{22}$ with no clear benefit in UTIs require hospitalization ${ }^{31}$ marked decrease in MRSA UTIS ${ }^{33}$ and CAUTIS $^{34}$ in a multi-intervention studies ${ }^{33,34}$ including contact precaution interventions

Encourage fluid intake/hydration to reduce infection Studied as single interventions ${ }^{24.26}$ and part of bundles 25 for the LTC setting with no significant benefits demonstrated regarding infection prevention Improve general patient hygiene to reduce infection Studied only as part of CAUTI bundles in the LTC setting including 1 with marked decreases in unspecified CAUTIs without statistical significance noted ${ }^{12}$ and $1^{20}$ without improvement in symptomatic UTIs

Cranberry product as prophylaxis The use of cranberry-containing products (eg, juice, capsules/tablets, extracts) has been assessed in recent systematic reviews and meta-analyses, $^{58,59,69}$ evaluating a total of 14 heterogeneous studies in multiple settings (outpatient, hospital, LTC, spinal cord injury). Both recent meta-analyses $^{56.59}$ demonstrated similar nonsignificant pooled risk ratios for symptomatic UTIs, although 1 meta-analysis found a significant protection for subgroups such as women with recurrent UTI ${ }^{59}$ that was seen in the other meta-analysis. ${ }^{58}$ Of note, individual studies in the LTC setting have

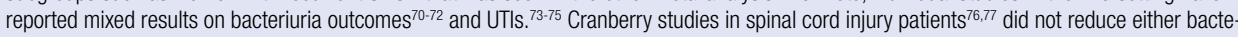
riuria or UTI outcomes. A very recent abstract ${ }^{78}$ regarding a double-blind placebo-controlled RCT published regarding effectiveness of twice daily cranberry capsules in LTC suggested reduced rates of clinically defined UTIs with treatment effect of $0.79(95 \% \mathrm{Cl}, 0.60-1.03)$ among patients at high risk for UTI (long-term catheterization, diabetes, $\geq 1$ UTI in prior year) and 0.83 (95\% Cl, 0.60-1.16) among patients at low risk for UTI, but not likely to be cost effective. ${ }^{79}$ In contrast, another very recently published double-blinded placebo-controlled RCT regarding the effectiveness of 2 oral cranberry capsules once daily resulted in no significant difference in the presence of bacteriuria plus pyruria over 1 year among older women residing in nursing homes. ${ }^{80}$

Vitamin/mineral supplement as UTI prophylaxis Ineffective in RCT ${ }^{81}$ for prevention of symptomatic UTIs per 1000 resident-days in LTC setting

Treatment of atrophic vaginitis as UTI prophylaxis Treatment of atrophic vaginitis with topical vaginal estrogens in postmenopausal women with recurrent UTIs (in outpatient setting) has been supported by RCTs (single blind ${ }^{82}$ and double-blind ${ }^{52}$ and by a respective chart review of a case series ${ }^{83}$ of female LTC nursing home residents with recurrent UTI.

Interventions to improve management of urinary Studied as educational strategies ${ }^{21,23,2,2,29,30,38,39}$ and protocols regarding incontinence care for staff and residents/family, in addition to interventions of incontinence specialists, ${ }^{23,39}$ providing individualized treatment plans to LTC residents, which can include a variety of interventions such as pelvic floor exercises, medical treatment for specific types of incontinence including avoidance of exacerbating medication and treatment of atrophic vaginitis

Implementation of effective infection control program Infection control program implementation often includes several interventions including hand-hygiene programs, and surveillance of nosocomial infections including UTI with the potential as feedback ${ }^{20}$ to motivate reductions in unnecessary catheter use and improved catheter care. Such interventions have been studied in the LTC setting in studies 20,21 including other specific interventions targeting CAUTIs (including infection control "walk rounds" for CAUTI detection, fed back daily to nurses). ${ }^{20}$

Interventions to Reduce Unnecessary Indwelling Urinary Catheter Placement:

Disrupting Lifecycle Stages 1 and 4 of Urinary Catheters

Education regarding the hazards of urinary catheters Educational interventions aiming to improve staff knowledge of CAUTI and urinary catheter risks are common components in multi-intervention studies implemented in both acute and LTC settings. Of note, in the LTC setting, educational strategies studied have included modules specific for all healthcare workers (unlicensed and licensed) who care for catheters with separate modules for nurses who insert catheters, with multiple formats including online, ${ }^{84,85}$ small-group teaching sessions and case reviews, and education of patients/residents ${ }^{13,25,29}$ and families. ${ }^{13,29}$

Education and/or policies regarding appropriate Education and policies regarding appropriate (and inappropriate) indications for indwelling catheters have been common in the acute care setting," indications for indwelling catheters often as part of a bundle of CAUTI preventive strategies, implementing the HICPAC list ${ }^{86}$ of appropriate indications. These lists have also been implemented in the LTC setting ${ }^{13,39}$ with either modifications of lists from acute care or LTC. ${ }^{87}$

Requiring physician order with appropriate indication Requiring physician orders for catheter placement has been studied in both acute care ${ }^{11}$ and LTC settings s.8. $^{13,84}$

before placing indwelling catheters

Requiring documentation of staff who insert the catheters with reason for catheter placement

Requiring nurses to document insertion with indication has been an intervention employed specifically in the emergency setting ${ }^{84}$ where catheters were placed without electronic orders and in settings where nurses are empowered to remove catheters by criteria

Education and supplies for alternatives to indwelling Facilitating use of alternatives to indwelling catheters is recommended ${ }^{86}$ and supported by either lower UTI or other complication rates in patients catheters such as external catheters, ISCs, and noncatheter strategies for managing incontinence

Urinary retention protocols for ISC and/or bladder scanner use before indwelling catheters requested treated with external catheters, ${ }^{36}$ intermittent catheters, and noncatheter ${ }^{88}$ strategies compared to indwelling Foley catheters

Interventions to Improve Catheter Insertion Technique:

Disrupting Lifecycle Stage 1 of Urinary Catheters

Education for aseptic insertion of indwelling catheters

Hands-on training/competency assessments regarding aseptic indwelling catheter insertion

Options regarding intermittent catheterization
Although not confirmed as effective by limited evidence, ${ }^{89}$ aseptic (as opposed to clean non-sterile) insertion of indwelling catheters is the accepted and recommended ${ }^{86,90}$ practice in all settings. Nurse education regarding urinary catheter avoidance, maintenance, insertion, and removal that included one-on-one teaching is preferred, and resulted in higher adherence to CAUTI prevention bundle elements over online education alone. ${ }^{91}$

Catheter placement by "only properly trained persons" using aseptic technique is recommended. ${ }^{86}$ The use of competency assessments in LTC has been studied $12,13,85$ in CAUTI bundles, although the individual impact of competency training interventions cannot be assessed from available studies. The CDC evidence-based guideline ${ }^{86}$ recommends that healthcare personnel and others who care for catheters be given periodic in-service training regarding techniques and procedures for catheter insertion, maintenance, and removal.

Clean vs. sterile, and single-use vs. multi-use intermittent catheterization has also been studied including several studies in the LTC and rehabilitation settings, ${ }^{92-95}$ with a systematic review60 indicating no evidence that UTI rates are impacted by these options, in agreement with evidence-based guideline ${ }^{86,90}$ indicating that clean (non-sterile) ISC is acceptable for patients requiring chronic ISC, with guidelines still recommending aseptic insertion for indwelling catheters, although the limited evidence ${ }^{89}$ regarding this is not convincing. 


\section{TABLE 3. Comprehensive List of Interventions Considered for Prevention of UTI and CAUTI (continued)}

Interventions

Standardizing catheter-placement supplies/kit

Catheter kit standardization (aiming to standardize catheter placement by making the necessary supplies readily available) is occurring in some acute care settings similar to prior "kit" interventions for prevention of blood-stream infections. Some LTC setting studies ${ }^{13}$ mention interventions regarding selection of catheter products but have not been specific regarding use of a catheter kit as opposed to individual catheter products.

Interventions to Improve Catheter Insertion Technique:

Disrupting Lifecycle Stage 1 of Urinary Catheters

Type of catheterization

Comparing different types of catheterization (indwelling catheters vs. ISCs vs. external catheters) has also been the subject of systematic reviews. $0 e^{62}$ systematic review had zero studies meeting the inclusion criteria. Another ${ }^{66}$ systematic review focused on suprapubic catheters, with the available evidence of 14 studies (no RCTs, 1 prospective nonrandomized study with a comparator, 8 retrospective reviews with comparators, a case series, and qualitative/descriptive assessments of quality of life) reports no evidence of differences between symptomatic UTI outcomes between suprapubic and urethral catheters, although the evidence is limited by varied UTI definitions applied for outcomes. However, a Cochrane systematic review $^{96}$ comparing short-term ( $<14$ days) of indwelling urethral urinary catheters to suprapubic urinary catheters found that groups with indwelling urinary catheters had more cases of bacteriuria (RR 2.6.95\% $\mathrm{Cl}, 2.12 .3 .18$ ) and significantly more patient discomfort (RR 2.98: 95\% $\mathrm{Cl}, 2.31$, 3.85). Evidence-based guidelines ${ }^{86}$ recommend ISC use is preferable to indwelling suprapubic or urethral catheters for bladder-emptying dysfunction, based on decreased rates of symptomatic UTIs and unspecified UTIs in select patient populations. Despite some evidence of lower CAUTI rates for external catheters and ISC compared to indwelling catheters, no catheter is preferable because of increased rates ${ }^{97,98}$ of symptomatic UTI even with nonindwelling catheters by observational studies.

Catheter coating/materials Different options in catheter coatings (such as hydrophilic-coated, antiseptic or antibiotic-impregnated) and materials (latex, PVC, silicone) have been studied.

Systematic reviews suggest either insufficient evidence for recommendation ${ }^{99}$ or no evidence that UTI rates are impacted by these options; the $\mathrm{CDC}^{86}$ targeted systematic review suggesting antimicrobial/aseptic catheters may be useful if CAUTI rates are not decreasing with other strategies. A more recent RCT in the acute care setting demonstrated no benefit of antimicrobial catheters. ${ }^{100}$

Although prior evidence-based guidelines were mixed ${ }^{86,90}$ regarding routine use of hydrophilic catheters for ISC, a 2013 systematic review and meta-analysis ${ }^{61}$ of hydrophilic catheters in the spinal cord injury population indicate these may be preferable (compared to standard nonhydrophilic catheters) for intermittent straight catheterization, with a significantly lower incidence of symptomatic or treated UTIs (OR, $0.36 ; 95 \% \mathrm{Cl}, 24 \%-54 \%$; $\mathrm{P}<0.001)$.

\begin{tabular}{ll}
\hline Catheter tip options & $\begin{array}{l}\text { Different options in catheter tip configurations for catheters used for intermittent catheterization (such as straight, coude, olive tip, or introducer-tip) } \\
\text { are discussed in narrative reviews citing potential benefits for certain patient populations (such as using coude catheters for men with enlarged } \\
\text { prostates). These types of recommendations may be valid clinically and are choices sometimes recommended by urologists in cases of difficult } \\
\text { placement. }{ }^{56} \text { There is insufficient evidence to recommend specific catheter tips as a general CAUTI bundle component for the average patient. }\end{array}$ \\
\hline Catheter size & The smallest bore catheter possible with consistent good drainage is recommended to avoid black neck and urethral mucosa trauma. ${ }^{54,86}$ \\
\hline Catheter length & $\begin{array}{l}\text { Narrative reviews suggest than the optimal catheter length varies by gender }{ }^{54} \text { ( } 45 \mathrm{~cm} \text {, males; } 25 \mathrm{~cm} \text {, females) to avoid kinking. Specific recommen- } \\
\text { dations regarding catheter length have not been provided by recent evidence-based reviews, although keeping the catheter free from kinking to } \\
\text { maintain unobstructed urine flow is recommended. }\end{array}$ \\
\hline "Clos,90
\end{tabular}

Maintenance/Care of Patients with Catheters:

Disrupting Lifecycle Stage 2 of Urinary Catheters

Handwashing, gloving before and after catheter/bag care

Keeping drainage bag below bladder

Irrigations, washouts, and instillations

Catheter replacement issues

Avoid equipment sharing between catheterized patients

Spatial separation of catheterized patients
Hand hygiene is recommended immediately before and after insertion or any manipulation of the urinary catheter or site. Gloves should be worn during any manipulation of catheterized patients or when providing intimate care. Gown use should be considered during catheter insertions, manipulation, and when providing assistance during activities of daily living. These strategies are useful regardless of a resident's colonization status with multidrug resistant organisms.

Keeping the collecting bag below the level of the bladder at all times without placement of the bag on the floor is recommended by evidence-based guidelines. ${ }^{86}$

Evidence-based guidelines ${ }^{86,90,101}$ recommend against cleaning the periurethral area with antiseptics to prevent CAUTI while the catheter is in place. Routine hygiene (cleansing of the meatal surface during daily bathing) is appropriate..$^{90}$

The practice of irrigating or washing out long-term indwelling urinary catheters has also been assessed by systematic reviews ${ }^{65,102}$ including reviews of various solutions (eg, saline, acidic solutions, antiseptic, and antibiotic solutions) have summarized 5 studies in multiple settings that were noted to be of poor quality and also did not appear to support these interventions as effective at either reductions of symptomatic CAUTIs or time to requiring first catheter change. Our own systematic search strategy identified several studies involving these interventions that either had been evaluated for the previously published systematic reviews (as included ${ }^{103,104}$ or excluded ${ }^{105}$ studies). Washout and irrigation strategies have also been assessed at length by a recent CDC-targeted systematic review ${ }^{86}$ with agreement that bladder irrigation and catheter drainage bag instillations are not recommended, given no differences in symptomatic UTI and mixed results in bacteriuria outcomes.

Catheter replacement at routine, fixed intervals is not recommended by evidence-based guidelines ${ }^{86}$ and did not decrease UTIs in the study reviewed in detail in this systematic review. ${ }^{35} \mathrm{~A}$ recent integrative review on catheter change intervals concluded there was insufficient evidence to support or refute the common practice of routine catheter changes but is a pre-emptive strategy employed in those who encrust and develop recurrent blockage. ${ }^{106}$

This has been recommended in narrative reviews ${ }^{45,107}$ and is reasonable and recommended by the $C D C$ guideline $e^{86}$ with regard to not sharing catheter-care supplies (such as devices used to empty catheter bags).

Spatial separation has been recommended by a case-control study, ${ }^{108}$ but further research is needed to assess the benefit of spatial separation of catheterized patients. ${ }^{86}$ 
TABLE 3. Comprehensive List of Interventions Considered for Prevention of UTI and CAUTI (continued)

Interventions

General Summary of Available Evidence and Recommendations Provided

Maintenance/Care of Patients with Catheters:

Disrupting Lifecycle Stage 2 of Urinary Catheters

Prophylaxis with systematic antimicrobials

The use of antimicrobial prophylaxis for chronically catheterized patients studied in several studies ${ }^{109-112}$ yielded by our search strategy has also been reviewed in a recent systematic review ${ }^{68}$ (of 8 studies, including indwelling catheters and ISCs) and systematic review and meta-analysis ${ }^{67}$ (0 15 studies involving ISCS) systematic reviews ${ }^{67,68}$ and meta-analyse ${ }^{67}$ with no benefit seen in patients with either chronic catheters or ISCs (with increased resistance ${ }^{67}$ suggested in ISC patients), in agreement with a recent $\mathrm{CDC}^{86}$ targeted systematic review. Our search did reveal a very recent study ${ }^{64}$ supporting the use of antimicrobial prophylaxis when short-term catheters are removed in the acute care setting; however, other studies indicate that prophylactic antimicrobials are not routinely indicated for changes of chronic catheters due to little morbidity ${ }^{45,113,114}$ reported with chronic catheter changes.

Other systemic chemoprophylaxis $\quad$ The evidence for methenamine IN preventing CAUTI is limited for use in both short-term catheterizations (studied only for postoperative gynecologic surgery) and long-term catheterizations, and not recommended for routine use for patients with long-term intermittent or long-term indwelling urethral or suprapubic catheterization according to evidence-based guidelines. ${ }^{86,90}$

Bacterial interference interventions

Novel interventions are being studied ${ }^{115}$ regarding the feasibility and potential benefit of "bacterial interference" interventions involving urinary colonization with benign bacteria, with the goal to reduce symptomatic infections by pathologic bacteria.

Prompting Removal of Unnecessary Catheters:

Disrupting Lifecycle Stage 3 of Urinary Catheters

Trial removal of indwelling catheters present at admission to LTC setting

This practice has been studied as a bundle component ${ }^{21,25,39}$ in LTC settings, and functions as a type of stop-order by prompting a trial removal of all indwelling catheters upon admission to LTC setting. This type of intervention may function similarly to stop-orders studied in the acute care setting. Studies reporting this type of intervention are advised to assess and report potential adverse events to patients, similar to acute care interventions using reminders and stop-orders. ${ }^{11}$

Urinary catheter reminders, reminding staff that

The use of reminders and/or stop-orders has been demonstrated by a recent systematic review and meta-analysis ${ }^{11}$ focused on the acute care patient/resident has a catheter to consider removing setting to reduce CAUTIs per 1000 catheter-days by more than 50\%; these studies often included reminders/stop-orders as part of a CAUTI

Urinary catheter stop-orders, requiring removal of catheter unless specific clinical criteria are met prevention bundle. Reminder types included use of daily checklists, electronic reminders, and the use of catheter patrols. Similar interventions have also been implemented in a few LTC studies including the use of catheter audit tools, ${ }^{39}$ daily assessment for continued catheter need, ${ }^{13}$ electronic removal reminder systems ${ }^{14}$ with some studies reporting decreased infections or catheter use, although most studies were underpowered to detect statistical significance of these interventions in the LTC setting.

NOTE: Abbreviations: CAUTI, catheter-associated urinary tract infection; CDC, Centers for Disease Control and Prevention; Cl, confidence interval; HICPAC, Healthcare Infection Control Practices Advisory Committee; ISC, intermittent straight catheterization; LTC, long-term care; OR, odds ratio; PVC, polyvinyl chloride; RCT, randomized controlled trial; RR, relative risk; UTI, urinary tract infection.

preemptive precautions for catheterized patients. ${ }^{34}$ Hydration was assessed in 3 studies. ${ }^{24-26}$

\section{Outcomes of Included Studies}

Table 2 describes the studies' outcomes reported for UTI, CAUTI, or bacteriuria. ${ }^{15,20-38}$ The outcome definitions of UTI and CAUTI varied widely. Only 2 studies $^{22,39}$ reported UTI outcomes using definitions specific for nursing home settings such as McGeer's criteria ${ }^{40}$ a detailed review and comparison of published CAUTI definitions used clinically and for surveillance in nursing homes is provided in Supplemental Table 3. Two studies reported symptomatic CAUTIs per 1000 catheter-days. ${ }^{32,34}$ Another study ${ }^{22}$ reported symptomatic CAUTIs per 1000 resident-days. Three reported symptomatic CAUTIs as counts..$^{35,38}$ Saint et $\mathrm{al}^{36}$ reported CAUTIs as part of a combined outcome (ie, bacteriuria, CAUTI, or death).

The 19 studies (Table 2) reported 12 UTI outcomes, ${ }^{15,20,21,23,25-31,33} 9$ CAUTI outcomes, ${ }^{15,22,32,34,35,38} 4$ bacteriuria outcomes, ${ }^{24,36,38}$ and 5 catheter use outcomes. ${ }^{21,29,30,37,38}$ Five studies showed CAUTI reduction ${ }^{15,22,32,34,35}$ (1 significantly $\left.^{34}\right) ; 9$ studies showed UTI reduction ${ }^{13,18,19,21,23-25,27,28,31}$ (none significantly); 2 studies showed bacteriuria reduction (none significantly). One study ${ }^{36}$ reported 2 composite outcomes including bacteriuria or CAUTI or death, with statistically significant improvement reported for 1 composite measure. Four studies reported catheter use, with all showing reduced catheter use in the intervention group; however, only 1 achieved statistically significant reduction. ${ }^{37}$

\section{Synthesis of Systematic Review Results}

Overall, many studies reported decreases in UTI, CAUTI, and urinary catheter use measures but without statistical significance, with many studies likely underpowered for our outcomes of interest. Often, the outcomes of interest in this systematic review were not the main outcome for which the study was designed and originally powered. The interventions studied included several currently implemented as part of CAUTI bundles in the acute care setting, such as improving catheter use, and care and infection control strategies. Other included interventions target common challenges specific to the nursing home setting such as removing indwelling catheters upon admission to the nursing home from an acute-care facility ${ }^{21,25}$ and applying interventions to address incontinence by either general strategies ${ }^{21,23,25,30,38}$ or the use of an incontinence specialist ${ }^{23}$ to provide individual treatment plans. The only intervention that demonstrated a statistically significant reduction in CAUTI in chronically catheterized patients employed a comprehensive program to improve antimicrobial use, hand hygiene (including hand hygiene and gloves for catheter care), and preemptive precautions for patients with devices, along with promotion of standardized CAUTI definitions and active multidrug resistant organism surveillance. ${ }^{34}$

\section{Narrative Review Results}

Table 3 includes a comprehensive list of potential interventions that have been considered for prevention of UTI or CAUTI (including those in acute care and nursing home 
settings), as summarized from this systematic review and prior narrative or systematic reviews. ${ }^{43-115}$

\section{DISCUSSION}

We performed a broad systematic review of strategies to decrease UTI, CAUTI, and urinary catheter use that may be helpful in nursing homes. While many studies reported decreased UTI, CAUTI, or urinary catheter use measures, few demonstrated statistically significant reductions perhaps because many were underpowered to assess statistical significance. Pooled analyses were not feasible to provide the expected impact of these interventions in the nursing home setting.

This review confirms that bundles of interventions for prevention ofCAUTI have been implemented with some evidence of success in nursing home settings, with several components in common with those implemented in the acute care setting, such as hand hygiene and strategies to reduce and improve catheter use. ${ }^{41}$ Some studies focused on issues more common in nursing homes such as chronic catheterization and incontinence. A nursing home CAUTI bundle should be designed with the resources and challenges present in the nursing home environment in mind, and with recognition that, although the number of patients with catheters is less than in acute care, there will be more patients with chronic catheterization needs and incontinence.

Although catheter utilization in nursing homes is low, further reductions in catheter days and CAUTIs can be achieved. Catheter removal reminders and stop orders have demonstrated a greater than $50 \%$ reduction in CAUTIs in acute care settings: ${ }^{11}$ an example of a stop-order intervention in nursing homes is trial removal of indwelling catheters present at facility admission without clear urologic need present at the time of admission. ${ }^{25}$ Nursing home interventions to avoid catheter placement should include incontinence programs, discussion of alternatives to indwelling urinary catheters with patients, families, and frontline personnel, and urinary retention protocols. Programs to reduce CAUTI should include education to improve aseptic insertion, and to maintain awareness and proper care of catheters in place by regular assessment of catheter necessity, securement, hand hygiene, and preemptive barrier precautions for catheterized patients. Interventions that focus on improving appropriate use of urine tests and antibiotics to treat UTIs can also significantly affect the rates of reported symptomatic CAUTIs, with the potential to decrease unnecessary antibiotic use..$^{20,21}$

The main limitation of this review is that many studies provided little information about their intervention and definition of outcomes. The strength of this review is the detailed and broad search strategy applied with generous inclusion of interventions and outcomes to highlight the available evidence and details of interventions that have been studied and implemented.

\section{CONCLUSION}

This review synthesizes the current state of evidence and proposes strategies to reduce UTIs in nursing homes. Inter- ventions that motivate catheter avoidance and catheter removal to prevent CAUTI in acute care ${ }^{11}$ and nursing home settings are supported by the strongest available evidence, although the strength of that evidence is less in the nursing home setting. Limitations notwithstanding, interventions such as incontinence care planning and hydration programs can reduce UTI in this population and is important for overall wellbeing.

\section{Acknowledgments}

The authors appreciate the guidance that Vineet Chopra MD, MSc, provided regarding options for methodological quality assessment tools, and the assistance of Mary Rogers $\mathrm{PhD}$, MS, in interpreting the published Downs and Black Quality Index items, which informed our modification of this tool for application in this study. The authors appreciate, also, the feedback provided by the Agency for Healthcare Research and Quality (AHRQ) Content and Materials Development Committee for the AHRQ Safety Program for Long-Term Care: Preventing CAUTI and other Healthcare-associated Infections.

Disclosures: Agency for Healthcare Research and Quality (AHRQ) contract \#HHSA290201000025I provided funding for this study, which was developed in response to AHRQ Task Order \#8 for ACTION II RFTO 26 CUSP for CAUTI in LTC. AHRQ developed the details of the task and provided comments on a draft report, which informed the report submitted to AHRQ in December 2013, used to inform the interventions for a national collaborative (http:/www.hret.org/quality/projects/ long-term-care-cauti.shtml). Dr. Meddings's effort on this project was funded by con current effort from her AHRQ (K08 HS19767). Dr. Saint's and Dr. Krein's effort on this project was funded by concurrent effort from the Veterans Affairs National Center for Patient Safety, Ann Arbor Patient Safety Center of Inquiry. Dr. Meddings's other research is funded by AHRQ (2R01HS018334-04), the NIH-LRP program, the VA National Center for Patient Safety, and the VA Ann Arbor Patient Safety Center of Inquiry. Dr. Krein's other research is funded by a VA Health Services Research and Development Award (RCS 11-222). Dr. Mody's other research is funded by VA Healthcare System Geriatric Research Clinical Care Center (GRECC), NIA-Pepper Center, NIA (R01AG032298, R01AG041780, K24AG050685-01). Dr. Saint has received fees for serving on advisory boards for Doximity and Jvion. All other authors report no financial conflicts of interest. The findings and conclusions in this report are those of the authors and do not necessarily represent those of the sponsor, the Agency for Healthcare Research and Quality, or the U.S. Department of Veterans Affairs. These analyses were presented in part as a poster presentation at the ID Week Annual Meeting on October 10, 2014 in Philadelphia, PA.

\section{References}

1. Beresford L. Post-acute patient care: new frontier for hospitalists. The Hospitalist. July 2015. http://www.the-hospitalist.org/hospitalist/article/122330/post-acute-patient-care-new-frontier-hospitalists. Accessed March 31, 2017.

2. Butterfield S. Hospital medicine matures: Hospitalists and hospitalist groups move into post-acute care. 2012. http://www.acphospitalist.org/archives/2012/10/ coverstory.htm. Accessed April 6, 2016.

3. Pittman D. SNFs: New Turf for Hospitalists? 2013. http://www.medpagetoday.com/ HospitalBasedMedicine/Hospitalists/39401. Accessed April 6, 2016.

4. Society of Hospital Medicine. SHM and IPC Healthcare Develop First SHM Primer for Hospitalists in Skilled Nursing Facilities. 2015. http://www.hospitalmedicine.org/Web/Media_Center/Press_Release/2015/SHM_and_IPC_Healthcare_Develop_First_SHM_Primer_for_Hospitalists_in_Skilled_Nursing_Facilities.aspx. Accessed April 6, 2016.

5. Montoya A, Mody L. Common infections in nursing homes: a review of current issues and challenges. Aging Health. 2011;7(6):889-899.

6. Phillips CD, Adepoju O, Stone N, et al. Asymptomatic bacteriuria, antibiotic use, and suspected urinary tract infections in four nursing homes. BMC Geriatr. 2012;12:73.

7. Rogers M, Mody L, Kaufman S, Fries B, McMahon L, Saint S. Use of urinary collection devices in skilled nursing facilities in five states. J Amer Geriatr Soc. 2008;56:854-861.

8. Castle N, Engberg JB, Wagner LM, Handler S. Resident and facility factors associated with the incidence of urinary tract infections identified in the nursing home minimum data set. J Appl Gerontol. 2015. doi: 10.1177/0733464815584666. 
9. Tsan L, Langberg R, Davis C, et al. Nursing home-associated infections in Department of Veterans Affairs community living centers. Am J Infect Control. 2010;38(6):461-466.

10. Kunin CM, Chin QF, Chambers S. Morbidity and mortality associated with indwelling urinary catheters in elderly patients in a nursing home--confounding due to the presence of associated diseases. J Am Geriatr Soc. 1987;35(11):1001 1006 .

11. Meddings J, Rogers MA, Krein SL, Fakih MG, Olmsted RN, Saint S. Reducing unnecessary urinary catheter use and other strategies to prevent catheter-associated urinary tract infection: an integrative review. BMJ Qual Saf. 2013;23(4):277-289.

12. Abraham F, Abraham FP. A CAUTI bundle with a twist. Am J Infect Control. 2012;40(5):e79-e80.

13. Flynn ER, Zombolis K. Reducing hospital acquired indwelling urinary catheter-associated urinary tract infections through multidisciplinary team and shared governance practice model. Am J Infect Control. 2011;39(5):E28-E29.

14. Gokula MR, Gaspar P, Siram R. Implementation of an evidence based protocol to reduce use of indwelling urinary catheters in the long term care environment. J Am Med Dir Assoc. 2013;14(3):B23.

15. Brownhill $\mathrm{K}$. Training in care homes to reduce avoidable harm. Nurs Times 2013;109(43):20-22.

16. Galeon CP, Romero I. Implementing a performance improvement project in a multi-level teaching facility on reducing catheter associated urinary tract infec tions (CAUTI). Am J Infect Control. 2014:S130-S131.

17. Evans ME, Kralovic SM, Simbartl LA, et al. Nationwide reduction of health care-associated methicillin-resistant Staphylococcus aureus infections in Veterans Affairs long-term care facilities. Am J Infect Control. 2014;42(1):60-62.

18. Evans KA, Ligon R, Lipton C. Reduction of antibiotic starts for asymptomatic bacteriuria in skilled nursing facilities. J Am Geriatr Soc. 2015;63:S131.

19. Downs SH, Black N. The feasibility of creating a checklist for the assessment of the methodological quality both of randomised and non-randomised studies of health care interventions. J Epidemiol Community Health. 1998;52(6):377-384.

20. Ahlbrecht H, Shearen C, Degelau J, Guay DR. Team approach to infection prevention and control in the nursing home setting. Am J Infect Control. 1999;27(1):64-70.

21. Cools HJ, van der Meer JW. Infection control in a skilled nursing facility: a 6-year survey. J Hosp Infect. 1988;12(2):117-124.

22. Fendler EJ, Ali Y, Hammond BS, Lyons MK, Kelley MB, Vowell NA. The impact of alcohol hand sanitizer use on infection rates in an extended care facility. Am J Infect Control. 2002;30(4):226-233.

23. Klay M, Marfyak K. Use of a continence nurse specialist in an extended care facility. Urol Nurs. 2005;25(2):101-102.

24. Lin S. A pilot study: fluid intake and bacteriuria in nursing home residents in southern Taiwan. Nurs Res. 2013;62(1):66-72.

25. McConnell J. Preventing urinary tract infections. Geriatr Nurs. 1984;5(8):361-362

26. Mentes JC, Culp K. Reducing hydration-linked events in nursing home residents. Clin Nurs Res. 2003;12(3):210-225; discussion 226-218.

27. Miller SC, Lepore M, Lima JC, Shield R, Tyler DA. Does the introduction of nursing home culture change practices improve quality? J Am Geriatr Soc. 2014;62(9):1675-1682.

28. Stuart RL, Orr E, Kotsanas D, Gillespie EE. A nurse-led antimicrobial stewardship intervention in two residential aged care facilities. Healthcare Infection. 2015;20(1):4-6

29. van Gaal B, Schoonhoven L, Mintjes JAJ, Borm GF, Koopmans RTCM, van Achterberg T. The SAFE or SORRY? programme. Part II: Effect on preventive care. Int J Nurs Stud. 2011;48(9):1049-1057.

30. van Gaal BGI, Schoonhoven L, Mintjes JAJ, et al. Fewer adverse events as a result of the SAFE or SORRY? programme in hospitals and nursing homes. part I: primary outcome of a cluster randomised trial. Int J Nurs Stud. 2011;48(9):1040-1048.

31. Yeung WK, Wilson Tam WS, Wong TW. Clustered randomized controlled trial of a hand hygiene intervention involving pocket-sized containers of alcohol-based hand rub for the control of infections in long-term care facilities. Infect Control Hosp Epidemiol. 2011;32(1):67-76.

32. Darouiche RO, Goetz L, Kaldis T, Cerra-Stewart C, AlSharif A, Priebe M. Impact of StatLock securing device on symptomatic catheter-related urinary tract infection: a prospective, randomized, multicenter clinical trial. Am J Infect Control. 2006;34(9):555-560.

33. Evans ME, Kralovic SM, Simbartl LA, et al. Prevention of methicillin-resistant Staphylococcus aureus infections in spinal cord injury units. Am J Infect Control. 2013;41(5):422-426.

34. Mody L, Krein S, Saint S, et al. A targeted infection prevention intervention in nursing home residents with indwelling devices: a randomized clinical trial. JAMA Intern Med. 2015;175:714-723.

35. Priefer BA, Duthie Jr EH, Gambert SR. Frequency of urinary catheter change and clinical urinary tract infection. Study in hospital-based, skilled nursing home. Urology. 1982;20(2):141-142.
36. Saint S, Kaufman SR, Rogers MA, Baker PD, Ossenkop K, Lipsky BA. Condom versus indwelling urinary catheters: a randomized trial. J Am Geriatr Soc. 2006;54(7):1055-1061

37. Suardi L, Cazzaniga M, Spinelli M, Tagliabue A. From intermittent catheterisation to time-volume dependent catheterisation in patients with spinal cord injuries, through the use of a portable, ultrasound instrument. Europa Medicophysica. 2001;37(2):111-114.

38. Tang MW, Kwok TC, Hui E, Woo J. Intermittent versus indwelling urinary catheterization in older female patients. Maturitas. 2006;53(3):274-281.

39. Cassel BG, Parkes V, Poon R, Rae H. Quality improvement best practices and long-term indwelling urinary catheters. Perspectives. 2008;32(1):13-17.

40. Stone ND, Ashraf MS, Calder J, et al. Surveillance definitions of infections in long-term care facilities: revisiting the McGeer criteria. Infect Control Hosp Epidemiol. 2012;33(10):965-977.

41. Saint S, Greene MT, Krein SL, et al. A Program to Prevent Catheter-Associated Urinary Tract Infection in Acute Care. New England Journal of Medicine. 2016;374(22):2111-2119.

42. McGeer A, Campbell B, Emori TG, et al. Definitions of infection for surveillance in long-term care facilities. Am J Infect Control. 1991;19(1):1-7.

43. Nicolle LE. The chronic indwelling catheter and urinary infection in long-termcare facility residents. Infect Control Hosp Epidemiol. 2001;22(5):316-321.

44. Nicolle LE; SHEA Long-Term Care Committee. Urinary tract infections in longterm-care facilities. Infect Control Hosp Epidemiol. 2001;22(3):167-175.

45. Nicolle LE. Catheter-related urinary tract infection. Drug $\mathcal{E}$ Aging. 2005;22(8):627-639.

46. Cochran S. Care of the indwelling urinary catheter - Is it evidence based? J Wound Ostomy Cont Nurs. 2007;34(3):282-288.

47. Seiler WO, Stahelin HB. Practical management of catheter-associated UTIs. Geriatrics. 1988;43(8):43-50.

48. Stickler DJ, Chawla JC. The role of antiseptics in the management of patients with long-term indwelling bladder catheters. J Hosp Infect. 1987;10(3):219-228.

49. Gray M. Does the construction material affect outcomes in long-term catheterization? J Wound Ostomy Cont Nurs. 2006;33(2):116-121.

50. Trautner BW, Darouiche RO. Clinical review: prevention of urinary tract infection in patients with spinal cord injury. J Spinal Cord Med. 2002;2002(25):277-283.

51. Maloney C. Estrogen \& recurrent UTI in postmenopausal women. Am J Nurs. 2002;102(8):44-52.

52. Raz R. Hormone replacement therapy or prophylaxis in postmenopausal women with recurrent urinary tract infection. J Infect Dis. 2001;183(suppl 1):S74-S76.

53. Godfrey H. Older people, continence care and catheters: dilemmas and resolutions. Br J Nurs. 2008;17(9):S4-S11.

54. Godfrey H, Evans A. Management of long-term urethral catheters: minimizing complications. Br J Nurs. 2000;9(2):74-76.

55. Kunin CM. Chemoprophylaxis and suppressive therapy in the management of urinary tract infections. J Antimicrob Chemother. 1994;33(suppl A):51-62.

56. Newman DK, Willson MM. Review of intermittent catheterization and current best practices. Urol Nurs. 2011;31(1):12-48.

57. Allan GM, Nicolle L. Cranberry for preventing urinary tract infection. Can Fam Physician. 2013;59(4):367.

58. Jepson RG, Williams G, Craig JC. Cranberries for preventing urinary tract infections. Cochrane Database Syst Rev. 2012;10:CD001321.

59. Wang $\mathrm{CH}$, Fang $\mathrm{CC}$, Chen NC, et al. Cranberry-containing products for prevention of urinary tract infections in susceptible populations: a systematic review and meta-analysis of randomized controlled trials. Arch Intern Med. 2012;172(13):988-996

60. Moore KN, Fader M, Getliffe K. Long-term bladder management by intermittent catheterisation in adults and children. Cochrane Database Syst Rev. 2007(4):CD006008

61. Li L, Ye WQ, Ruan H, Yang BY, Zhang SQ. Impact of hydrophilic catheters on urinary tract infections in people with spinal cord injury: systematic review and meta-analysis of randomized controlled trials. Arch Phys Med Rehabil. 2013;94(4):782-787.

62. Jamison J, Maguire S, McCann J. Catheter policies for management of long term voiding problems in adults with neurogenic bladder disorders. Cochrane Database Syst Rev. 2011(12):CD004375.

63. Gray M. What nursing interventions reduce the risk of symptomatic urinary tract infections in the patient with an indwelling catheter? J Wound Ostomy Cont Nurs. 2004:31(1):3-13

64. Marschall J, Carpenter C, Fowler S, Trautner B. Antibiotic prophylaxis for urinary tract infections after removal of urinary catheter: meta-analysis. BMJ. 2013;346:f3147.

65. Sinclair L, Hagen S, Cross S. Washout policies in long-term indwelling urinary catheterization in adults: a short version Cochrane review. Neurourol Urodyn. 2011;30(7):1208-1212 
66. Hunter KF, Bharmal A, Moore KN. Long-term bladder drainage: suprapubic catheter versus other methods: a scoping review. Neurourol Urodyn. 2013;32(7):944-951.

67. Morton SC, Shekelle PG, Adams JL, et al. Antimicrobial prophylaxis for urinary tract infection in persons with spinal cord dysfunction. Arch Phys Med Rehabil 2002;83(1):129-138.

68. Niël-Weise BS, van den Broek PJ, da Silva EM, Silva LA. Urinary catheter policies for long-term bladder drainage. Cochrane Database Syst Rev. 2012(8).

69. Jepson R, Craig J. Cranberries for preventing urinary tract infections. Cochrane Database Syst Rev. 2008;10(CD001321).

70. Avorn J, Monane M, Gurwitz JH, Glynn RJ, Choodnovskiy I, Lipsitz LA. Reduction of bacteriuria and pyuria after ingestion of cranberry juice. JAMA. 1994:271(10):751-754.

71. Bianco L, Perrelli E, Towle V, Van Ness PH, Juthani-Mehta M. Pilot randomized controlled dosing study of cranberry capsules for reduction of bacteriuria plus pyuria in female nursing home residents. J Am Geriatr Soc. 2012;60(6):1180-1181

72. Lin SC, Wang CC, Shih SC, Tjung JJ, Tsou MT, Lin CJ. Prevention of Asymptomatic Bacteriuria with Cranberries and Roselle Juice in Home-care Patient with Long-term Urinary Catheterization. Int J Gerontol. 2014;8(3):152-156.

73. Juthani-Mehta M, Perley L, Chen S, Dziura J, Gupta K. Feasibility of cranberry capsule administration and clean-catch urine collection in long-term care residents. J Am Geriatr Soc. 2010;58(10):2028-2030.

74. Tully CL, Bastone P, Vaughan J, Ballentine L. Urinary tract infection prophylaxis with cranberry extract in the nursing home setting. J Am Geriatr Soc. 2004;52(4):S206-S206

75. Woodward N. Use of cranberry extract for the prevention of UTIs in an at-risk population. 41st Annual Wound, Ostomy and Continence Nurses Annual Con ference, St. Louis, Missouri, June 6-10, 2009. J Wound Ostomy Continence Nurs. 2009;36(3S):S62-S62

76. Linsenmeyer TA, Harrison B, Oakley A, Kirshblum S, Stock JA, Millis SR. Evaluation of cranberry supplement for reduction of urinary tract infections in individuals with neurogenic bladders secondary to spinal cord injury. A prospective, double-blinded, placebo-controlled, crossover study. J Spinal Cord Med. 2004;27(1):29-34.

77. Waites KB, Canupp KC, Armstrong S, DeVivo MJ. Effect of cranberry extract on bacteriuria and pyuria in persons with neurogenic bladder secondary to spinal cord injury. J Spinal Cord Med. 2004;27(1):35-40.

78. Caljouw MAA, Van Den Hout WB, Putter H, Achterberg WP, Cools HJM Gussekloo J. Effectiveness of cranberry capsules to prevent urinary tract infections in vulnerable older persons. A double-blind randomized placebo-controlled trial in long-term care facilities. Eur Geriatr Med. 2013;4:S118-S119.

79. Hout WB, Caljouw MAA, Putter H, Cools HJM, Gussekloo J. Cost-effectiveness of cranberry capsules to prevent urinary tract infection in long-term care facilities: economic evaluation with a randomized controlled trial. J Am Geriatr Soc. 2014;62(1):111-116.

80. Juthani-Mehta M, Van Ness PH, Bianco L, et al. Effect of cranberry capsules on bacteriuria plus pyuria among older women in nursing homes: A randomized clinical trial. JAMA. 2016;316(18):1879-1887.

81. Liu BA, McGeer A, McArthur MA, et al. Effect of multivitamin and mineral supplementation on episodes of infection in nursing home residents: a randomized, placebo-controlled study. J Am Geriatr Soc. 2007;55(1):35-42.

82. Eriksen B. A randomized, open, parallel-group study on the preventive effect of an estradiol-releasing vaginal ring (Estring) on recurrent urinary tract infections in postmenopausal women. Am J Obstet Gynecol. 1999;180:1072-1079.

83. Maloney C. Hormone replacement therapy in female nursing home residents with recurrent urinary tract infection. Ann Long-Term Care. 1998;6(3):77-82.

84. Gokula RM, Smith MA, Hickner J. Emergency room staff education and use of a urinary catheter indication sheet improves appropriate use of foley catheters. Am J Infect Control. 2007;35(9):589-593

85. Salamon L. Catheter-associated urinary tract infections: a nurse-sensitive indicator in an inpatient rehabilitation program. Rehabil Nurs. 2009;34(6):237-241.

86. Gould CV, Umscheid CA, Agarwal RK, Kuntz G, Pegues DA. Guideline for prevention of catheter-associated urinary tract infections 2009. Infect Control Hosp Epidemiol. 2010;31(4):319-326.

87. American Medical Directors Association (AMDA). Appropriate indications for use of a chronic indwelling catheter in the long-term care setting. Columbia, MD; excerpted from AMDA's Clinical Practice Guideline: Urinary Incontinence. 2005.

88. Rannikko S, Kyllastinen M, Granqvist B. Comparison of long-term indwelling catheters and bed-pads in the treatment of urinary incontinence in elderly patients. J Infect. 1986;12(3):221-227.

89. Carapeti EA, Andrews SM, Bentley PC. Randomised study of sterile versus non-sterile urethral catheterization. Ann R Coll Surg Engl. 1996;78(1):59-60.

90. Hooton TM, Bradley SF, Cardenas DD, et al. Diagnosis, prevention, and treat- ment of catheter-associated urinary tract infection in adults: 2009 International Clinical Practice Guidelines from the Infectious Diseases Society of America. Clin Infect Dis. 2010;50(5):625-663

91. Olsen-Scribner RJ, Hayes C, Pottinger P. Sustaining reduction of catheter-associated urinary tract infection (CAUTI)-outcomes after two educational methods in a regional university-affiliated medical center. Am J Infect Control. 2014;1:S22.

92. Duffy LM, Cleary J, Ahern S, et al. Clean intermittent catheterization: safe, cost-effective bladder management for male residents of VA nursing homes. J Am Geriatr Soc. 1995;43(8):865-870.

93. Joseph C, Jacobson C, Strausbaugh L, Maxwell M, French M, Colling J. Sterile vs clean urinary catheterization. J Am Geriatr Soc. 1991;39(10):1042-1043.

94. Moore KN, Burt J, Voaklander DC. Intermittent catheterization in the rehabilitation setting: a comparison of clean and sterile technique. Clin Rehabili. 2006;20(6):461-468.

95. Moore KN, Kelm M, Sinclair O, Cadrain G. Bacteriuria in intermittent catheterization users: the effect of sterile versus clean reused catheters. Rehabil Nurs J. 1993;18(5):306-309.

96. Niel-Weise BS, van den Broek PJ. Urinary catheter policies for short-term bladder drainage in adults. Cochrane Database Syst Rev. 2005(3):CD004203.

97. Ouslander JG, Greengold B, Chen S. External catheter use and urinary tract infections among incontinent male nursing home patients. J Am Geriatr Soc. 1987;35(12):1063-1070

98. Wyndaele JJ, Brauner A, Geerlings SE, Bela K, Peter T, Bjerklund-Johanson TE. Clean intermittent catheterization and urinary tract infection: review and guide for future research. BJU Int. 2012;110(11 Pt C):E910-917.

99. Jahn P, Beutner K, Langer G. Types of indwelling urinary catheters for long-term bladder drainage in adults. Cochrane Database Syst Rev. 2012(10):CD004997.

100. Pickard R, Lam T, Maclennan G, et al. Antimicrobial catheters for reduction of symptomatic urinary tract infection in adults requiring short-term catheterisation in hospital: a multicentre randomised controlled trial. Lancet. 2012;380(9857):1927-1935.

101. Burke JP, Garibaldi RA, Britt MR, Jacobson JA, Conti M, Alling DW. Prevention of catheter-associated urinary tract infections. Efficacy of daily meatal care regimens. Am J Med. 1981;70(3):655-658

102. Hagen S, Sinclair L, Cross S. Washout policies in long-term indwelling urinary catheterisation in adults. Cochrane Database Syst Rev. 2010(3).

103. Moore KN, Hunter KF, McGinnis R, et al. Do catheter washouts extend patency time in long-term indwelling urethral catheters? A randomized controlled trial of acidic washout solution, normal saline washout, or standard care. J Wound Ostomy Continence Nurs. 2009;36(1):82-90.

104. Muncie HL Jr, Hoopes JM, Damron DJ, Tenney JH, Warren JW. Once-daily irrigation of long-term urethral catheters with normal saline. Lack of benefit. Arch Intern Med. 1989;149(2):441-

105. Ruwaldt MM. Irrigation of indwelling urinary catheters. Urology. 1983;21(2):127-129.

106. Palka MA. Evidenced based review of recommendations addressing the frequency of changing long-term indwelling urinary catheters in older adults. Geriatr Nurs. 2014;35(5):357-363.

107. Warren JW. Catheter-associated urinary tract infections. Infect Dis Clin North Am. 1997;11(3):609-622.

108. Fryklund B, Haeggman S, Burman LG. Transmission of urinary bacterial strains between patients with indwelling catheters--nursing in the same room and in separate rooms compared. J Hosp Infect. 1997;36(2):147-153.

109. Anderson RU. Non-sterile intermittent catheterization with antibiotic prophylaxis in the acute spinal cord injured male patient. J Urol. 1980;124(3):392-394.

110. Anderson RU. Prophylaxis of bacteriuria during intermittent catheterization of the acute neurogenic bladder. J Urol. 1980;123(3):364-366.

111. Gribble MJ, Puterman ML. Prophylaxis of urinary tract infection in persons with recent spinal cord injury: a prospective, randomized, double-blind, placebo-controlled study of trimethoprim-sulfamethoxazole. Am J Med. 1993;95(2):141-152.

112. Rutschmann OT, Zwahlen A. Use of norfloxacin for prevention of symptomatic urinary tract infection in chronically catheterized patients. Eur J Clin Microbiol Infect Dis. 1995;14(5):441-444.

113. Jewes LA, Gillespie WA, Leadbetter A, et al. Bacteriuria and bacteraemia in patients with long-term indwelling catheters--a domiciliary study. J Med Microbiol. 1988;26(1):61-65

114. Warren JW, Damron D, Tenney JH, Hoopes JM, Deforge B, Muncie HL, Jr. Fever, bacteremia, and death as complications of bacteriuria in women with long-term urethral catheters. J Infect Dis. 1987;155(6):1151-1158.

115. Prasad A, Cevallos ME, Riosa S, Darouiche RO, Trautner BW. A bacterial interference strategy for prevention of UTI in persons practicing intermittent catheterization. Spinal Cord. 2009;47(7):565-569. 